\title{
Research on Application of SDN in Dispatching System of Power Grid YAN Zijian ${ }^{1, \text { a }}$, ZHU Guiying ${ }^{1}$,LIU Xiaomei ${ }^{1}$,SUN Peng ${ }^{1}$ \\ ${ }^{1}$ China Electric Power Research Institute, Nanjing 210003, Jiangsu Province, China ayanzijian@epri.sgcc.com.cn
}

Keywords: SDN; dispatching data network; fine-grained controlling level; Qos of end-to-end; network monitoring.

Abstract: With rapid development of power grid, the accurate and centralized analysis are characteristic of the future dispatching trend in power grid, all information of dispatching system at all level need to be shared. The demand for optimization operation of dispatching data network is more and more urgent. SDN is a new flexible frame for dispatching data network that can be more flexible in controlling network flow. This paper analysis feasibility of application about SDN applied in dispatching data network, and prompt typical application scenarios. Based on SDN the new architecture of the dispatching data network is proposed for network optimization and flexible control. The service flow can be classified by more fine-grained controlling level than before. Qos of end-to-end technology and network monitoring technology is elaborated in this paper, Above all, SDN can bring broad a widely prospect of application in dispatching data network in power grid.

\section{Introduction}

Today, SDN's influence has been swept up by a variety of large and small data centers, has also been extended to the wide area network. SDN can be regarded as a revolutionary IT wave after personal computer, internet, cloud computing. SDN can bring the benefit that includes centralized network control, flexible programming, reducing the hardware cost, simple operation and maintenance. These advantages determine that SDN has a wide application prospect in power system.

With the construction of the rapid development of interconnected power grid scheduling and UHVDC data network, it greatly enhances resources allocation capability through power optimization. Power grid operation face many challenges, these challenges includes how to promote centralized monitoring level, global decision-making ability, the allocation capability of resources in dispatching plan and new energy consumptive capacity. The situation put forward higher requirements than before, all information of dispatching system at all level need to be shared. At the base of sharing data, we can realize centralized analysis and control in dispatching system.

Above all, the demand for optimization operation of dispatching data network is more and more urgent. SDN is a new flexible frame to network operation in IT, which can change network operation by software defined network technology. Whether the SDN can be used for scheduling system, and how it is applied in the scheduling system, the current research is still in the primary stage. This paper focus on the feasibility of application about SDN applied in scheduling data network, and prompt typical application scenarios.

\section{The Software Defined Networking Framework}

The SDN architecture is shown as Fig.1.Software-Defined Networking (SDN) is an emerging architecture that is dynamic, manageable, cost-effective, and adaptable, making it ideal for the high-bandwidth, dynamic nature of today's applications. This architecture decouples the network control and forwarding functions enabling the network control to become directly programmable and the underlying infrastructure to be abstracted for applications and network services.

All SDN models have some version of an SDN Controller, as well as southbound APIs and northbound APIs:

Controllers:The "brains" of the network, SDN Controllers offer a centralized view of the overall network, and enable network administrators to dictate to the underlying systems (like 
switches and routers) how the forwarding plane should handle network traffic.

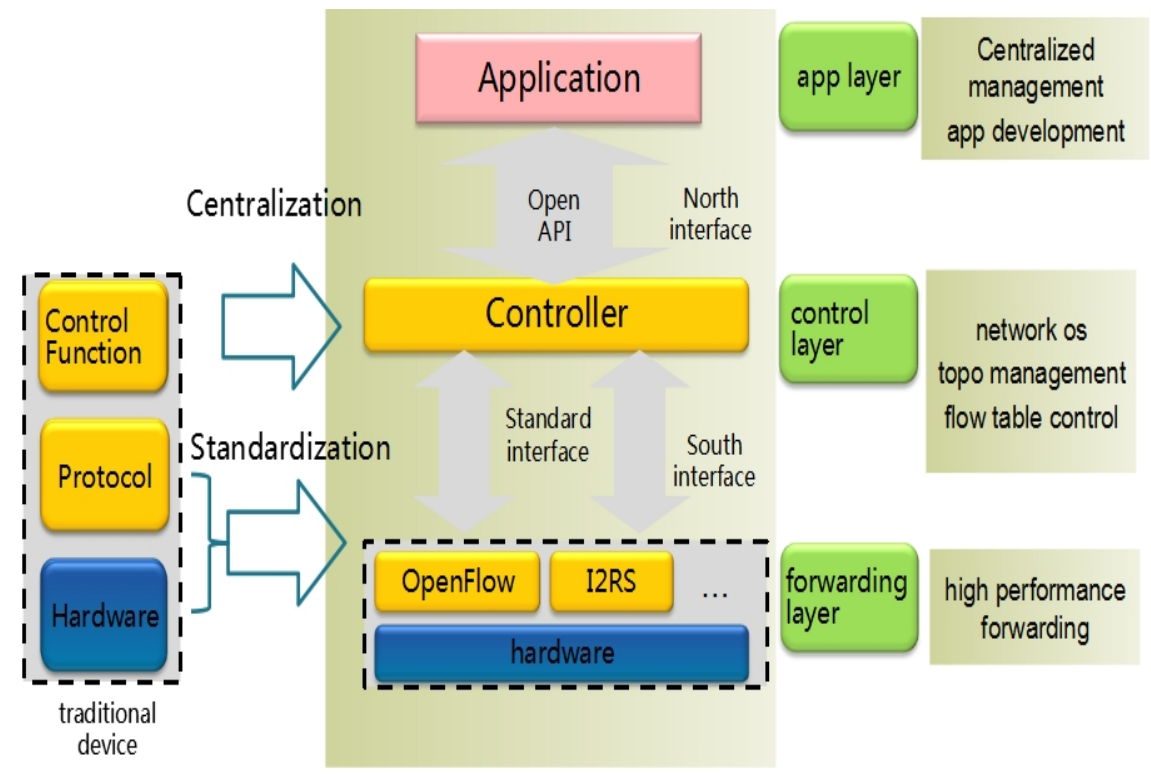

Fig 1 SDN architecture

I Southbound APIs:Software-defined networking uses southbound APIs to relay information to the switches and routers "below." OpenFlow, considered the first standard in SDN, was the original southbound API and remains as one of the most common protocols. Despite some considering OpenFlow and SDN to be one in the same, OpenFlow is merely one piece of the bigger SDN landscape.

I Northbound APIs: Software Defined Networking uses northbound APIs to communicate with the applications and business logic "above." These help network administrators to programmatically shape traffic and deploy services.

The SDN architecture offer a centralized, programmable network that can dynamically provision so as to address the changing needs of businesses, software-define networking, also provides the following benefits:

I Directly Programable: Network directly programmable because the control functions are decoupled from forwarding functions, which enable the network to be programmatically configured by proprietary or open source automation tools, including OpenStack,Puppet, and Chef.

I Centralized Management: Network intelligence is logically centralized in SDN controller software that maintains a global view of the network, which appears to applications and policy engines as a single, logical switch.

I Deliver Agility and Flexibility: Software Defined Networking helps organizations rapidly deploy new applications, services, and infrastructure to quickly meet changing business goals and objectives.

I Enable Innovation:SDN enables organizations to create new types of applications, services, and business models that can offer new revenue streams and more value from the network.

\section{Feasibility applied in dispatching system}

National Grid Corporation launched the "overall framework of new generation grid dispatching technology support system and key technology research" technology project, the project proposed "physical distribution, logical unification" mind to realize the overall structure, which includes the business architecture, hardware and software architecture, service architecture and maintenance 
architecture. The overall mechanism of real-time data processing based on "wide-area storage, distributed processing, global sharing, unified access" mind is established. On this basis, the whole integrated application analysis and decision center is implemented to achieve the accuracy about analysis results. The basic data comes from dispatch center at all level, so it must be obtained through dispatch data network, the network flow of dispatch data network may be much larger than in the past. In addition, integrated analysis and decision-making center AGC and other advanced applications of the analysis results need to be sent to all levels of scheduling Center, the final regulation of the corresponding power plant generator output of the movements in the control of information transmission can not have a delay, or affect the regulation of the results and grid operation.

\section{Current situation of dispatching data network}

The national grid dispatching data network (SGDnet, hereinafter referred to as "dispatching data network"), which was put into operation in 2003, is a data network for power dispatching production. It realizes real-time production between dispatching centers and dispatching centers Data transmission and exchange infrastructure. Currently, the dispatching data network provides two VPNs, namely real-time VPN and non-real-time VPN for the scheduling service, which carry the security area I service and the security area II service, respectively. The security area I service includes real-time data communication between EMS and RTU or power station automation system, real-time data exchange between EMS, WAMS data acquisition and stability control system, etc. The security area II service includes tie line exchange plan, tie line assessment, electric energy metering information, GPS data, DTS anti-accident system data. The existing dispatching data network can realize the real-time production data transmission and exchange demand between the dispatching center and the substation.

Dispatching network frame is shown as Fig.2.

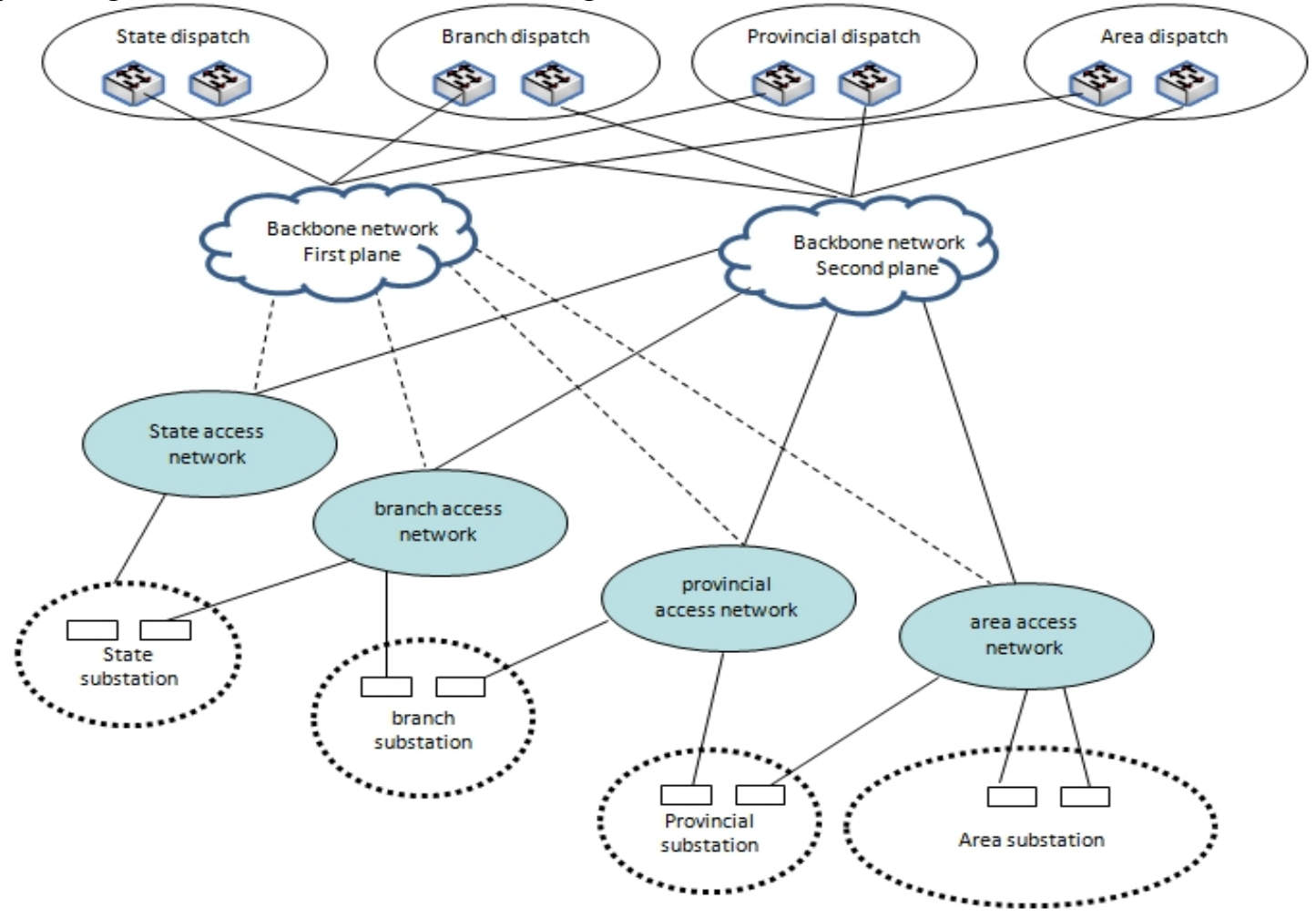

Fig.2 traditional dispatching network frame 


\section{Advantage of SDN}

Compared to traditional mode of network, SDN applied dispatching system can bring many advantages: (1) the operation status of network can be centralized monitoring, reason of overload flow in network can be analysized and suggestions of optimization of network planning can be provided; (2) SDN frame provide greater granularity to distinguish packet, classify important packet and normal packet for following Qos;(3)Through centralized management of network device, Qos control can achieved for end to end;(4)It is convenient to maintenance through centralized management of network device.(5)Through agile programming load-balance can be realized and the utilization of network bandwidth can be improved. In addition, the SDN frame can effectively adapt to the demand for new service.

\section{Dispatching data network Based on SDN}

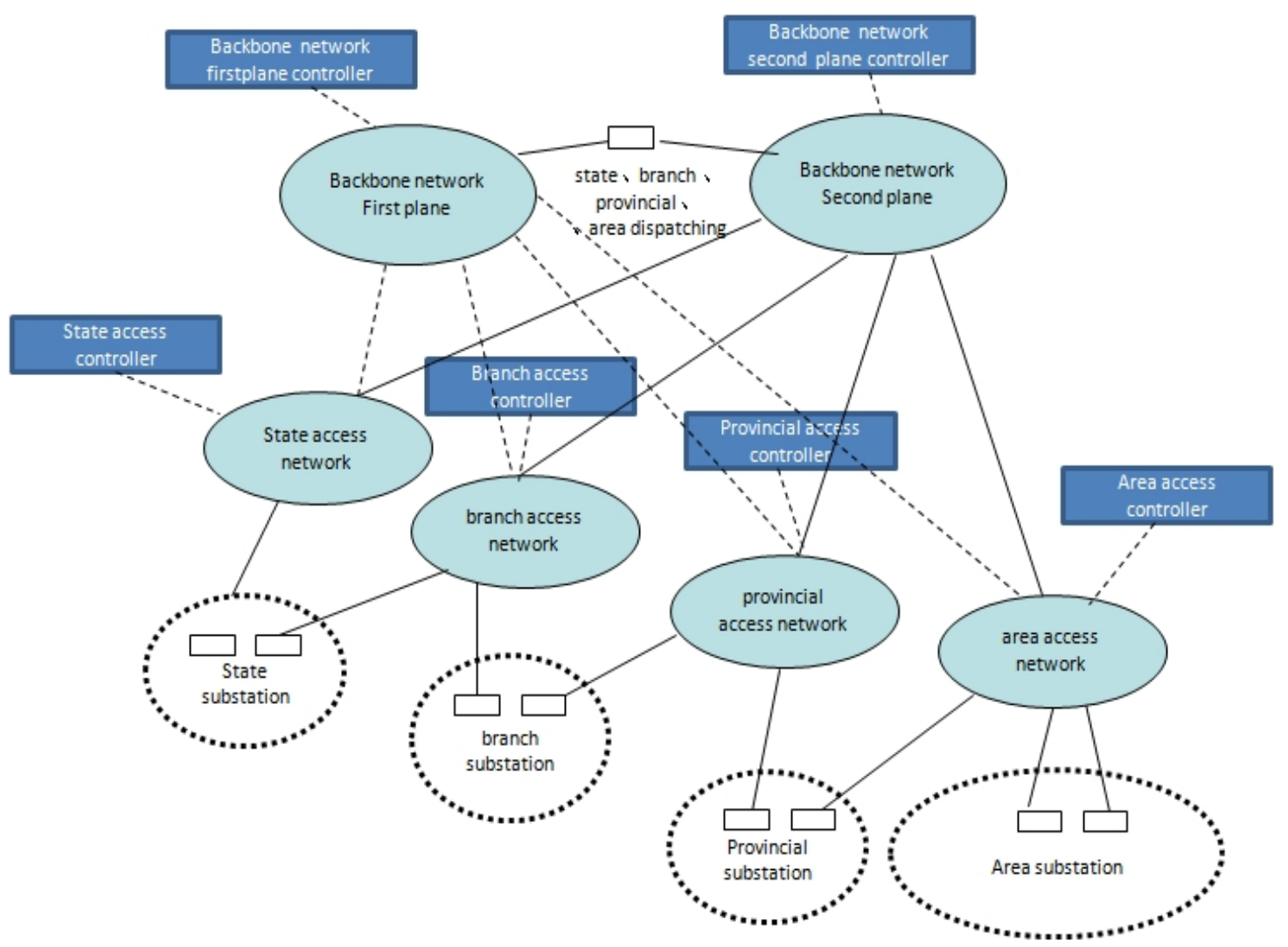

Fig.3 dispatching network frame based on SDN

The dispatching network frame based on SDN is shown as Fig.3. Due to adopt SDN frame, the switching equipment no longer need to calculate the routing information. The routing computation work transfer to SDN controller. So if the network range controlled by SDN controller is too big, the time cost of computation for routing is also big. Considering the quick computation of routing, we divided the network according autonomous domain.

According to the division of the autonomous domain, the backbone network of first plane (including state, backup state, branch dispatching) is controlled by single controller. Every access network (including state access network, branch access network, provincial access network, area access network) also adopt single controller. 


\section{Service Data and Qos Demand}

Table.1 Service Data List

\begin{tabular}{c|l|c|c|c}
\hline Security area & \multicolumn{1}{|c|}{ type } & priority & times & reliability \\
\hline \multirow{5}{*}{ I area } & Dispatching real-time data & high & sec & high \\
\cline { 2 - 5 } & WAMS data & high & sec & high \\
\cline { 2 - 5 } & Real-time market data & high & sec & high \\
\cline { 2 - 5 } & Area centralized monitoring data & high & sec & high \\
\cline { 2 - 5 } & Five Prevention & high & sec & high \\
\hline \multirow{5}{*}{ II area } & Tie line data & normal & day & high \\
\cline { 2 - 5 } & Plan data, load forecasting & normal & day & high \\
\cline { 2 - 5 } & dispatching operation order & high & random & high \\
\cline { 2 - 5 } & Water dispatching real-time data & high & sec & high \\
\hline
\end{tabular}

Import packet of dispatching network includes remote sign, remote measure, remote control, remote adjustment packet.

\section{Qos of end-to-end service}

It is important to ensure transmission reliability of important packet such as remote control packet etc.

In tradition network, it is difficult to realize Qos of end-to-end service due to distributed control. SDN can effectively realize Qos of end-to-end service by centralizing control.

Qos process is shown as Fig.3. We divide network flow into important flow and normal flow. Important flow is protected by Qos, normal flow select a relative optimal path. There are three advantages through Qos strategy. Firstly, the important packet is protected for required bandwidth, and best path is selected for it. Secondly, there are sub-optimal path for normal packet. Thirdly, the network usage rate is promoted greatly. 


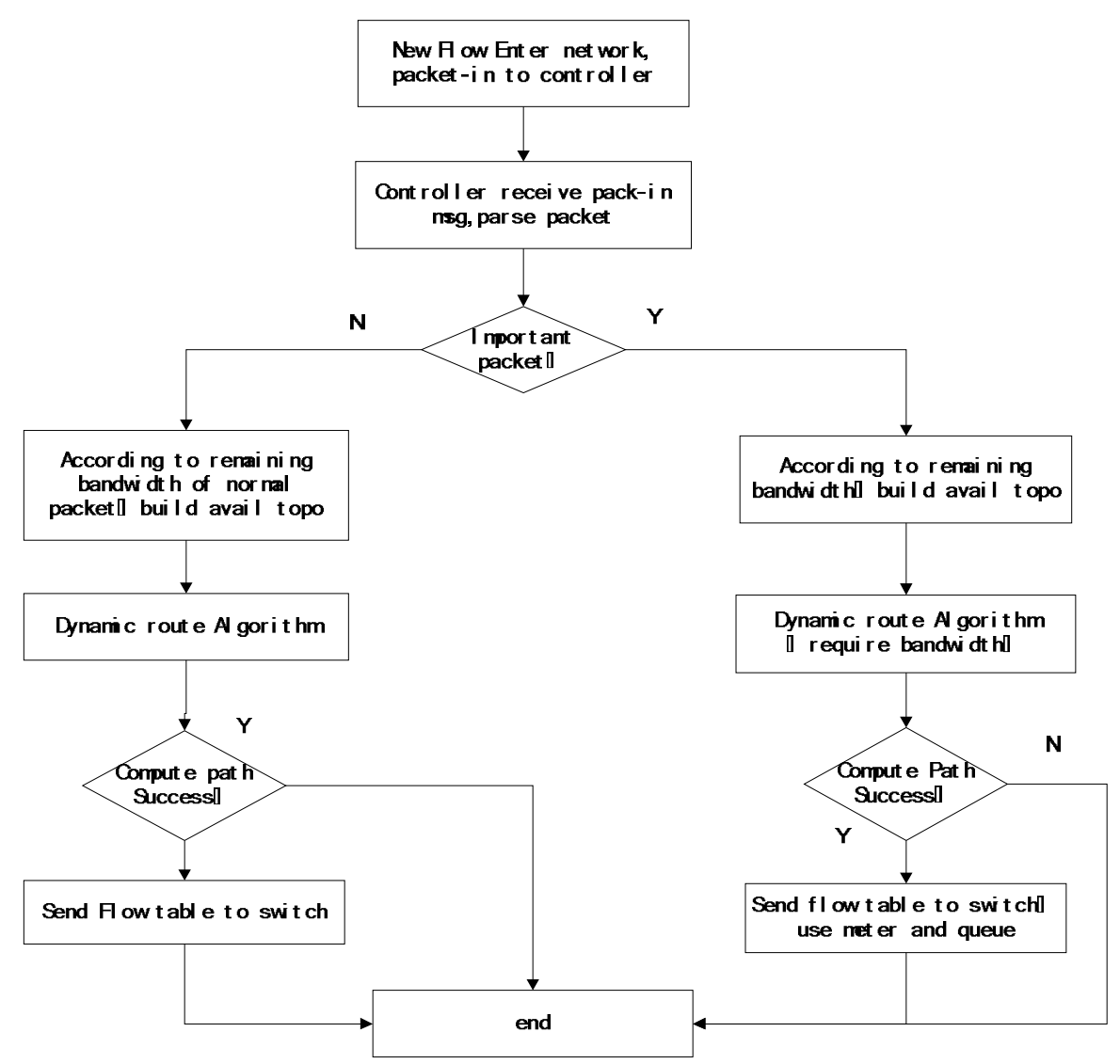

Fig.3 Qos process

\section{Network monitoring based on SDN}

With the growing scale and importance of the network, network monitoring and measurement has attracted much attention.

There are two key technologies to be researched, one is auto sense of network topology, one is network flow monitoring.

\section{Auto sense of network topology}

Auto sense of Network topology is the base of network management.

The link discovery is that controller use south-bound interface to communicate with the equipment for integrated monitoring and statistical reporting information. Link discovery technology is the key to obtain information of the whole network, and is based on the network address learning, VLAN, routing and forwarding network function. Link discovery of SDN network is finished by controller.

SDN controller mainly uses LLDP (Layer Discovery Protocol Link, Link Layer Discovery Protocol) as a link discovery protocol, which provides a standard link discovery method,

The controller needs to send LLDP packet as packet-out message timely, and obtain information by receiving packet-in message from switch directly connected, then complete the discovery function of topology construction, and complete updates of network topology when monitoring the working state of the switch. 


\section{Bandwidth measurement}

Obtaining the existing resources and status of the network is an important prerequisite for all subsequent work.

bandwidth data is an important data in the network state. In the SDN network bandwidth can be obtained from OpenFlow protocol, and also can be obtained from the third party measurement software, such as sFlow. Here is only the introduction of how to get the available bandwidth through the OpenFlow protocol.

The bandwidth of a link is determined by the capacity of the two ports. So we can get the network flow of the link by getting the flow of the port. The statistical message includes as the port, flow table, flow table entry, group table and meter table, which can be obtained from OpenFlow protocol. Taking the port statistics as an example, the controller can get the statistics information of the switch port through the period of the statistics Port message.

From the message format, you can find the number of packets to send and receive, the number of bytes, and the duration of this statistic. If we subtract the statistical number of bytes from two different time packet, then divided by time difference, statistical flow velocity can be computed. If you want to get the remaining bandwidth, you can subtract the maximum bandwidth with current flow bandwidth of port, you can get the remaining bandwidth of port.

\section{Link delay measurement}

There are no link delay information from OpenFlow protocol, we can obtain link delay from indirect method. The topology of link delay measurement is shown as Fig.4, which include one SDN controller and two OpenFlow switches.

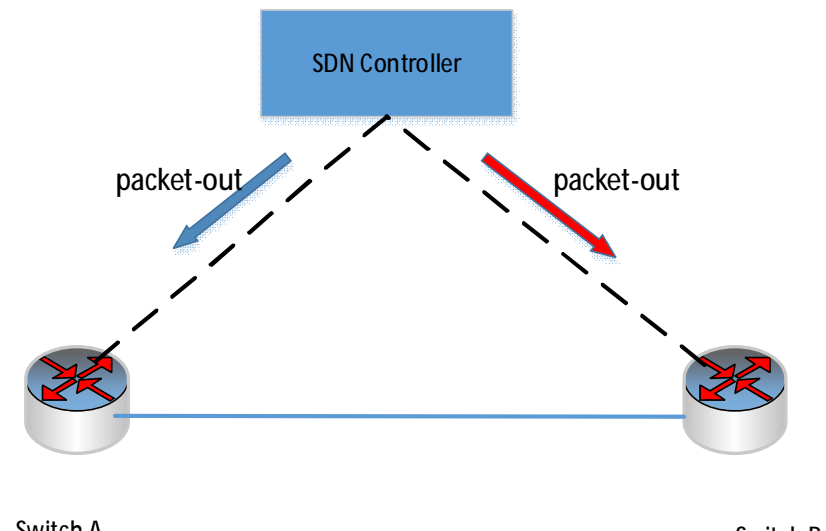

Fig.4 delay measurement of link

\section{Conclusion}

The Development of SDN is important revolution of IT, which can impact on dispatching data network from now on. At this situation, we need to research feasibility of application and design in dispatching system for flexible network management.

This paper research the feasibility of application about SDN applied in scheduling data network, and prompt typical application scenarios, key technologies about SDN are researched in detail.

With the development of SDN and urgent demand for dispatching network, There are broad application prospect in dispatching network. 


\section{Acknowledgements}

This work was financially supported by State Grid Corporation of China (DZ71-16-030).

\section{References}

[1] LIU Li-rong, WANG Yu-dong, XIAO Zhi-hong, LIU Ying, YAN Pei-li, XIN Pei-zhe, Discuss on the Construction Scheme of Power Dispatching Data Network, Telecommunications for Electric Power System , 2011 , 32(220) , 18-21

[2] WU Peng, WU Jun-min, LIU Chuan, et al. Research on the application of software defined network in electric power data communication network[J]. Information Technology, 2014(1):52-55.

[3] Open Networking Foundation. OpenFlow switch specification version 1. 3. 4[R]. 2014

[4] Open Networking Foundation. OpenFlow management and configuration protocol, OF-CONFIG 1. 2[R]. 2014

[5] YANG Shengchun, TANG Biqiang, YAO Jianguo , et al .Architecture and Key Technologies for Situational Awareness Based Automatic Intelligent Dispatching of Power Grid , Power System Technology , 2014 , 38(1), 33-39 .

[6] YAO , Jianguo , LAI Yening. The Essential Cause and Technical Requirements of the Smart Grid . Automation of Electric Power Systems , 2010 , 34(2) , 1-4 .

[7] YAO Jianguo, YAN Sheng, YANG Shengchun, et al . Practice and prospects of intelligent dispatch with Chinese characteristics [ J ]. Automation of Electric Power Systems , 2009 , 33(17) : $16-20$. 